\title{
QUADRATIC SPLINE COLLOCATION METHOD FOR WEAKLY SINGULAR INTEGRAL EQUATIONS AND CORRESPONDING EIGENVALUE PROBLEM $^{1}$
}

\author{
R. PALLAV and A. PEDAS
}

Institute of Applied Mathematics, University of Tartu

Liivi 2, 50409 Tartu, Estonia

E-mail: rene.pallav@ut.ee arvet.pedas@ut.ee

\section{ABSTRACT}

A quadratic spline collocation method for the numerical solution of weakly singular Fredholm integral equations of the second kind and corresponding eigenvalue problem is constructed. Using quasi-uniform and special graded grids, the rate of convergence of proposed numerical schemes is studied theoretically and numerically.

Key words: weakly singular integral equations, collocation method, quadratic splines

\section{INTRODUCTION}

We consider a linear integral equation

$$
\lambda y(t)=\int_{a}^{b} K(t, s) y(s) d s+f(t), \quad a \leq t \leq b, \lambda \neq 0,
$$

with $-\infty<a<b<\infty$ and given functions $K$ and $f$. We assume that

$$
K(t, s)=g(t, s) \kappa(t-s),
$$

where $g$ is a 3 times continuously differentiable function on $[a, b] \times[a, b]$ and $\kappa$ is a 2 times continuously differentiable function on $[a-b, b-a] \backslash\{0\}$ such that the estimate

$$
\left|\kappa^{\prime \prime}(\tau)\right| \leq c|\tau|^{-\beta} \quad(c=\text { const, } 0<\beta<3)
$$

\footnotetext{
${ }^{1}$ This work was supported by the Estonian Science Foundation (Research Grant No. 4410).
} 
holds with a $\beta \in(0,3)$ for every $\tau \in[a-b, b-a] \backslash\{0\}$. In the following by $c, c_{0}, c_{1}, \ldots$ we will denote positive constants, which may be different in different inequalities. It is clear from (1.3) that the following upper bounds for $\kappa^{\prime}$ and $\kappa$ can be derived: if $\beta \in(0,3) \backslash\{1,2\}$ then

$$
\left|\kappa^{(j)}(\tau)\right| \leq c_{j}\left(|\tau|^{-\beta+2-j}+1\right), \quad \tau \in[a-b, b-a] \backslash\{0\}, j=0,1 ;
$$

if $\beta=1$ then

$$
\left|\kappa^{\prime}(\tau)\right| \leq c_{1}(|\ln | \tau||+1), \quad|\kappa(\tau)| \leq c_{0}, \tau \in[a-b, b-a] \backslash\{0\} ;
$$

if $\beta=2$ then

$$
\left|\kappa^{\prime}(\tau)\right| \leq c_{1}\left(|\tau|^{-1}+1\right), \quad|\kappa(\tau)| \leq c_{0}(|\ln | \tau||+1), \tau \in[a-b, b-a] \backslash\{0\} .
$$

Thus, if $2 \leq \beta<3$, then the kernel $K(t, s)$ of equation (1.1) may have a weak singularity at $t=s$; if $0<\beta<2$ then $K$ is bounded but its derivatives may be singular. Integral equations with kernels of this type arise in the potential theory, atmospheric physics, and many other fields.

About the forcing function $f$ in equation (1.1) we will assume that $f \in$ $C^{3, \beta}[a, b]$, where

$$
C^{3, \beta}[a, b]=\left\{y \in C[a, b] \cap C^{3}(a, b): \sup _{a<t<b} \frac{\left|y^{\prime \prime \prime}(t)\right|}{(t-a)^{-\beta}+(b-t)^{-\beta}}<\infty\right\} .
$$

Here by $C[a, b]$ the Banach space of continuous functions $y(t), t \in[a, b]$, with the norm $\|y\|_{C[a, b]}=\max _{a \leq t \leq b}|y(t)|$ is denoted and $C^{3}(a, b)$ is the set of all 3 times continuously differentiable functions $y(t), t \in(a, b)$. Notice that $C^{3, \beta}[a, b], 0<\beta<3$, is a Banach space with respect to the norm

$$
\|y\|_{C^{3, \beta}[a, b]}=\|y\|_{C[a, b]}+\sup _{a<t<b} \frac{\left|y^{\prime \prime \prime}(t)\right|}{(t-a)^{-\beta}+(b-t)^{-\beta}}, \quad y \in C^{3, \beta}[a, b] .
$$

Note also that the inclusion $y \in C^{3, \beta}[a, b]$ implies the following inequalities for $y^{\prime}, y^{\prime \prime}$ and $y^{\prime \prime \prime}$ : if $\beta \in(0,3) \backslash\{1,2\}$ then

$$
\left|y^{(j)}(t)\right| \leq c_{j}\left[(t-a)^{-\beta+3-j}+(b-t)^{-\beta+3-j}\right], \quad t \in(a, b), j=1,2,3
$$

if $\beta=1$ then for $y^{\prime \prime \prime}$ the estimate (1.7) holds and

$$
\left|y^{\prime \prime}(t)\right| \leq c_{2}[|\ln (t-a)|+|\ln (b-t)|+1], \quad\left|y^{\prime}(t)\right| \leq c_{1}, \quad t \in(a, b) ;
$$

if $\beta=2$ then for $y^{\prime \prime}$ and $y^{\prime \prime \prime}$ the estimates (1.7) hold and

$$
\left|y^{\prime}(t)\right| \leq c_{1}[|\ln (t-a)|+|\ln (b-t)|+1], \quad t \in(a, b) .
$$

The regularity properties of a solution $y$ of equation (1.1) are stated by 
Theorem 1.1 (see [19], p. 7). Let the assumptions (1.2) and (1.3) hold. Let $f \in C^{3, \beta}[a, b]$ and let equation (1.1) have an integrable solution $y$. Then $y \in C^{3, \beta}[a, b]$.

Under the hypothesis of Theorem 1.1 the derivatives of a solution of equation (1.1) are, in general, unbounded near the boundary points $a$ and $b$ of the domain of integration. Therefore it is quite complicated to construct high order approximation methods for the numerical solution of such equations. In the sequel we construct for equation (1.1) and for corresponding eigenvalue problem

$$
\lambda y(t)=\int_{a}^{b} K(t, s) y(s) d s, \quad a \leq t \leq b,
$$

a collocation method using continuously differentiable quadratic splines on quasi-uniform and special graded grids. We will discuss the convergence rate of the proposed schemes and present some numerical illustrations. Our discussion is based on the corresponding results of $[7 ; 12 ; 13]$, where the case of non-homogeneous equations (1.1) is considered. Using some ideas and results of $[8-10 ; 17 ; 19]$, we also derive error estimates for the approximate eigenvalues of problem (1.10). Finally, we refer to $[1-6 ; 11 ; 14 ; 16 ; 18]$, where a discussion about the numerical solution of integral equations with weakly singular or other nonsmooth kernels by the collocation method is given.

Remark 1.1. In Theorem 1.1 we do not suppose that equation (1.1) is uniquely solvable. If $f=0$, then the statement of Theorem 1.1 yields the estimates (1.7) - (1.9) for the derivatives of the eigenfunctions of the integral operator $T$ defined by the formula

$$
(T y)(t)=\int_{a}^{b} K(t, s) y(s) d s, \quad a \leq t \leq b .
$$

\section{QUADRATIC SPLINE COLLOCATION METHOD}

For $n \in \mathbb{N}=\{1,2, \ldots\}$ let

$$
\Delta_{n}=\left\{t_{0}, t_{1}, \ldots, t_{n}: a=t_{0}<t_{1}<\ldots<t_{n}=b\right\}
$$

be a grid on $[a, b]$ (a partition of the interval $[a, b]$ with grid points $t_{i} \equiv t_{i}^{(n)}$, $i=0,1, \ldots, n)$. We say that the grid $\Delta_{n}$ is quasi-uniform if

$$
\max _{0 \leq i \leq n-1}\left(t_{i+1}-t_{i}\right) / \min _{0 \leq i \leq n-1}\left(t_{i+1}-t_{i}\right) \leq q,
$$

where $q \geq 1$ is a fixed real number not depending on $n$. We say that $\Delta_{n}$ is a graded grid if

$$
\left\{\begin{array}{l}
t_{i}=a+\frac{b-a}{2}\left(\frac{2 i}{n}\right)^{r}, \quad i=0,1, \ldots, n / 2 \\
t_{n / 2+i}=a+b-t_{n / 2-i}, \quad i=1,2, \ldots, n / 2
\end{array}\right.
$$


where $n=2 k, k \in \mathbb{N}$, and $r \geq 1$ is a fixed real number not depending on $n$. Here the parameter $r$ characterizes the nonuniformity of the grid: if $r=1$ then we obtain a uniform grid, if $r>1$ then the grid points $t_{0}, t_{1}, \ldots, t_{n}$ are more densely clustered near the endpoints $a$ and $b$ of the interval $[a, b]$. Notice that for $r>1$ the graded grid is not quasi-uniform.

Let $S_{2,1}\left(\Delta_{n}\right)$ be the set of quadratic splines with defect 1 on the grid (2.1):

$$
S_{2,1}\left(\Delta_{n}\right)=\left\{z \in C^{1}[a, b]:\left.z\right|_{\left[t_{i}, t_{i+1}\right]} \in \pi_{2}, i=0,1, \ldots, n-1\right\} .
$$

Here $\pi_{2}$ denotes the set of all polynomials of degree not exceeding 2 and $C^{1}[a, b]$ is set of all continuously differentiable functions $y(t), t \in[a, b]$. Let

$$
x_{0}=a ; \quad x_{i}=t_{i-1}+\eta\left(t_{i}-t_{i-1}\right), \quad i=1,2, \ldots, n ; \quad x_{n+1}=b,
$$

where $\eta \in(0,1)$ is a fixed real number not depending on $n$.

For given $n \in \mathbb{N}$ we look for an approximation $y_{n}$ to the solution $y$ of equation (1.1) in the linear space $S_{2,1}\left(\Delta_{n}\right)$ determining $y_{n} \in S_{2,1}\left(\Delta_{n}\right)$ from the conditions

$$
\lambda y_{n}\left(x_{i}\right)=\int_{a}^{b} K\left(x_{i}, s\right) y_{n}(s) d s+f\left(x_{i}\right), \quad i=0,1, \ldots, n+1 .
$$

Note that the conditions (2.6) determine a system of linear equations which exact form depends on the choice of a basis in the space $S_{2,1}\left(\Delta_{n}\right)$. For example, we can seek the approximation $y_{n} \in S_{2,1}\left(\Delta_{n}\right)$ in the form

$$
y_{n}(t)=\sum_{i=0}^{n+1} c_{i} B_{2, i}(t), \quad t \in[a, b]
$$

where $\left\{c_{i}\right\}$ are constants to be determined and $\left\{B_{2, i}\right\}$ is the B-spline basis in the space $S_{2,1}\left(\Delta_{n}\right)$ (cf. [15]):

$$
B_{2, i}(t)=\left\{\begin{array}{l}
\left(t-t_{i-2}\right)^{2} /\left(t_{i}-t_{i-2}\right)\left(t_{i-1}-t_{i-2}\right), t \in\left[t_{i-2}, t_{i-1}\right) \\
\frac{\left(t-t_{i-2}\right)\left(t_{i}-t\right)}{\left(t_{i}-t_{i-2}\right)\left(t_{i}-t_{i-1}\right)}+\frac{\left(t_{i+1}-t\right)\left(t-t_{i-1}\right)}{\left(t_{i+1}-t_{i-1}\right)\left(t_{i}-t_{i-1}\right)}, t \in\left[t_{i-1}, t_{i}\right) \\
\left(t_{i+1}-t\right)^{2} /\left(t_{i+1}-t_{i-1}\right)\left(t_{i+1}-t_{i}\right), t \in\left[t_{i}, t_{i+1}\right) \\
0, \text { otherwise, }
\end{array}\right.
$$

with $i=0,1, \ldots, n$, and

$$
B_{2, n+1}(t)=\left\{\begin{array}{l}
\left(t-t_{n-1}\right)^{2} /\left(t_{n}-t_{n-1}\right)^{2}, t \in\left[t_{n-1}, t_{n}\right] \\
0, \text { otherwise }
\end{array}\right.
$$


Here $t_{-2}=t_{-1}=t_{0}, t_{n+2}=t_{n+1}=t_{n}, t_{i} \in \Delta_{n}, i=0,1, \ldots, n$. Now the collocation conditions $(2.6)$ yield an $(n+2) \times(n+2)$ linear system with respect to unknowns $c_{0}, c_{1}, \ldots, c_{n+1}$; once these unknowns have been found, the approximation $y_{n}$ can be composed by formula (2.7).

Theorem 2.1 (see [7; 13]). Let the assumptions (1.2) and (1.3) hold and let $f \in C^{3, \beta}[a, b]$. Let the homogeneous integral equation (1.10), corresponding to the equation (1.1) by $f=0$, have only the trivial solution $y=0$. Finally, let the interpolation points (2.5) with the quasi-uniform grid $\{(2.1),(2.2)\}$ or the graded grid $\{(2.1),(2.3)\}$ be used.

Then equation (1.1) has a unique solution $y \in C[a, b]$ and for all sufficiently large $n \in \mathbb{N}$, say $n \geq n_{0}$, the collocation conditions (2.6) determine a unique approximation $y_{n} \in S_{2,1}\left(\Delta_{n}\right)$ to $y$. For $n \geq n_{0}$ the following error estimate holds:

$$
\left\|y_{n}-y\right\|_{C[a, b]} \leq c \delta_{n}
$$

where $c$ is a positive constant not depending on $n$ and

$$
\delta_{n}=n^{3-\beta}
$$

if the quasi-uniform grid $\{(2.1)),((2.2)\}$ is used, and

$$
\delta_{n}=\left\{\begin{array}{lr}
n^{-r(3-\beta)}, & 1 \leq r \leq 3 /(3-\beta) \\
n^{-3}, & r>3 /(3-\beta)
\end{array}\right.
$$

if the graded grid $\{(2.1),(2.3)\}$ is used.

\section{EIGENVALUE PROBLEM}

We consider eigenvalue problem (1.10), where the kernel $K$ has the form (1.2). For given $n \in \mathbb{I}$ we construct an approximating eigenvalue problem to (1.10) in the form

$$
\lambda y_{n}\left(x_{i}\right)=\int_{a}^{b} K\left(x_{i}, s\right) y_{n}(s) d s, \quad i=0,1, \ldots, n+1
$$

where $y_{n} \in S_{2,1}\left(\Delta_{n}\right)$ and the interpolation points $\left\{x_{i}\right\}$ are defined by (2.5). Choosing a basis in the space $S_{2,1}\left(\Delta_{n}\right)$ and presenting $y_{n}$ as a linear combination of basis functions, (3.1) will take the form of finite dimensional eigenvalue problem of linear algebra. In particular, we may use the presentation (2.7) with the basis functions $\{(2.8),(2.9)\}$.

For a Banach space $E$ we denote by $\mathcal{L}(E)$ the Banach space of all linear bounded operators $A: E \rightarrow E$ with the norm

$$
\|A\|_{\mathcal{L}(E)}=\sup \{\|A x\|: x \in E,\|x\|=1\} .
$$


For the operator $T$ defined by (1.10) it follows from (1.2) - (1.6) that $T \in$ $\mathcal{L}(C[a, b])$ and $T$ is compact as an operator from $C[a, b]$ to $C[a, b]$. We consider equation (1.10) as the operator equation $\lambda y=T y$ in the Banach space $C[a, b]$. Whereas $T: C[a, b] \rightarrow C[a, b]$ is compact, all eigenvalues of $T$ form a discrete set in the complex plane $\boldsymbol{C}$ with zero as the only possible limit and every point $\lambda_{0} \neq 0$ of the spectrum $\sigma(T)$ of $T$ is an isolated eigenvalue of $T$ with finite algebraic multiplicity. Recall that a complex number $\lambda_{0}$ is called an eigenvalue of $T$ if there exists an element $y^{0} \in C[a, b], y^{0} \neq 0$, such that $T y^{0}=\lambda_{0} y^{0}$; the element $y^{0}$ is called an eigenelement (eigenfunction) of $T$. For every nonzero eigenvalue $\lambda_{0}$ of $T$ both the eigenspace

$$
N\left(\lambda_{0} I-T\right)=\left\{y \in C[a, b]:\left(\lambda_{0} I-T\right) y=0\right\}
$$

and generalized eigenspace

$$
W=W\left(\lambda_{0}, T\right)=N\left(\left(\lambda_{0} I-T\right)^{\gamma}\right)
$$

of $T$ corresponding to the eigenvalue $\lambda_{0}$ are finite dimensional. Here $I$ is the identity mapping in the space $C[a, b]$ and $\gamma=\gamma\left(\lambda_{0}, T\right)$ is the rank of the eigenvalue $\lambda_{0}$, that is, the least positive integer such that

$$
N\left(\left(\lambda_{0} I-T\right)^{\gamma}\right)=N\left(\left(\lambda_{0} I-T\right)^{\gamma+1}\right) .
$$

The dimension $\mu$ of $W$ is called the algebraic multiplicity of $\lambda_{0}: \mu=$ $\mu\left(\lambda_{0}, T\right)=\operatorname{dim} W\left(\lambda_{0}, T\right)$.

Further, we consider eigenvalue problem (3.1) as the operator equation

$$
\lambda y_{n}=P_{n} T y_{n}
$$

in the Banach space $C[a, b]$. Here $P_{n}: C[a, b] \rightarrow C[a, b]$ is an interpolation operator which assigns to every function $y \in C[a, b]$ its piecewise quadratic interpolation function $P_{n} y \in S_{2,1}\left(\Delta_{n}\right) \subset C[a, b]$ satisfying the conditions $\left(P_{n} y\right)\left(x_{i}\right)=y\left(x_{i}\right), i=0,1, \ldots, n+1$. For both the quasi-uniform grid $\{(2.1),(2.2)\}$ and the graded grid $\{(2.1)(2.3)\}$ we obtain (see [7; 13]) that $P_{n} \in \mathcal{L}(C[a, b])$ implying $P_{n} T \in \mathcal{L}(C[a, b]), n \in \mathbb{N}$.

Theorem 3.1. Let the assumptions (1.2) and (1.3) hold and let the interpolation nodes (2.5) with grid points $\{(2.1),(2.2)\}$ or $\{(2.1),(2.3)\}$ be used.

Then for every nonzero eigenvalue $\lambda_{0}$ of $T$ (of equation (1.10)) there exists a sequence $\left\{\lambda_{n}\right\}$ of eigenvalues $\lambda_{n}$ of $P_{n} T$ (of equations (3.1)) such that $\lambda_{n} \rightarrow$ $\lambda_{0}$ for $n \rightarrow \infty$. Conversely, if $\left\{\lambda_{n}\right\}$ is a sequence of eigenvalues $\lambda_{n}$ of $P_{n} T$, and $\lambda_{0} \neq 0$ is an accumulation point for $\left\{\lambda_{n}\right\}$, then $\lambda_{0}$ is an eigenvalue of $T$.

Proof. On the basis of $[7 ; 13]$ we obtain that for both the quasi-uniform grid $\{(2.1),(2.2)\}$ and the graded grid $\{(2.1),(2.3)\}$,

$$
\left\|P_{n}\right\|_{\mathcal{L}(C[a, b])} \leq c_{1}, \quad n=1,2, \ldots,
$$


and

$$
\left\|y-P_{n} y\right\|_{C[a, b]} \leq c_{2} \delta_{n} \quad \forall y \in C^{3, \beta}[a, b], 0<\beta<3,
$$

where $c_{1}$ and $c_{2}$ are some positive constants not depending on $n$, and $\delta_{n}$ is given by $(2.11)$ or $(2.12)$, corresponding to the grid $\{(2.1),(2.2)\}$ or $\{(2.1),(2.3)\}$, respectively. Since the space $C^{3}[a, b]$ of all 3 times continuously differentiable functions on $[a, b]$ belongs to $C^{3, \beta}[a, b]$, we have according to $(3.3)$ that

$$
\left\|y-P_{n} y\right\|_{C[a, b]} \leq c_{2} \delta_{n} \quad \forall y \in C^{3}[a, b]
$$

Therefore, $\left\|y-P_{n} y\right\|_{C[a, b]} \rightarrow 0$ if $n \rightarrow \infty$ for every $y \in C^{3}[a, b]$. Since $C^{3}[a, b]$ is dense in $C[a, b]$, we have by (3.2) and Banach-Steinhaus theorem that $\left\|y-P_{n} y\right\|_{C[a, b]} \rightarrow 0$, if $n \rightarrow \infty$ for every $y \in C[a, b]$. From this and the compactness of the operator $T \in \mathcal{L}(C[a, b])$ we obtain

$$
\left\|T-P_{n} T\right\|_{\mathcal{L}(C[a, b])} \rightarrow 0, \quad n \rightarrow \infty
$$

Now the assertions of Theorem 3.1 follow from general results about the convergence of approximate eigenvalues, proved under the assumptions of regular or compact approximation of operators (see, for example, [8;9;17]). In particular, we can apply Theorem 5.1 from [19], p. 68 .

Remark 3.1. Let $\Lambda_{0}$ be some compact in the complex plane $\mathbb{C}$ so that $0 \notin \Lambda_{0}$ and $\lambda_{0}$ is the unique eigenvalue of $T$ in $\Lambda_{0}$. It follows from Theorem 3.1 that for all sufficiently large $n \in \mathbb{N}$, say $n \geq n_{0}$, at least one eigenvalue $\lambda_{n}$ of $P_{n} T$ belongs to $\Lambda_{0}$. Moreover, on the basis of (3.4) we obtain (cf. [19], p. 68) that the sum of the algebraic multiplicities $\mu\left(\lambda_{n}, P_{n} T\right)$ of all eigenvalues $\lambda_{n}$ of $P_{n} T$ in $\Lambda_{0}$ is equal to the algebraic multiplicity $\mu\left(\lambda_{0}, T\right)$ of the eigenvalue $\lambda_{0}$ of $T$ :

$$
\sum_{\lambda_{n}: \lambda_{n} \in \sigma\left(P_{n} T\right) \cap \Lambda_{0}} \mu\left(\lambda_{n}, P_{n} T\right)=\mu\left(\lambda_{0}, T\right), \quad n \geq n_{o}
$$

Theorem 3.2. Let the assumptions of Theorem 3.1 be fulfilled. Let $\lambda_{0} \neq 0$ be an eigenvalue of $T$ (of equation (1.10)) and let $\Lambda_{0} \subset \boldsymbol{C}$ be some compact, such that $0 \notin \Lambda_{0}$ and $\lambda_{0} \in \Lambda_{0}$ is the unique eigenvalue of $T$ in $\Lambda_{0}$. Finally, let $\left\{\lambda_{n}\right\}$ be a sequence of eigenvalues $\lambda_{n}$ of $P_{n} T$ (of equations (3.1)), such that $\lambda_{n} \rightarrow \lambda_{0}$ for $n \rightarrow \infty$.

Then for all sufficiently large $n \in I N$, say $n \geq n_{0}$, the following estimates hold:

$$
\left|\lambda_{n}-\lambda_{0}\right| \leq c_{1} \delta_{n}^{1 / \gamma}, \quad\left|\lambda_{n}-\lambda_{0}\right| \leq c_{2} \delta_{n}^{1 / k_{n}}, \quad\left|\bar{\lambda}_{n}-\lambda_{0}\right| \leq c_{3} \delta_{n}
$$

Here $c_{1}, c_{2}$ and $c_{3}$ are some positive constants not depending on $n, \gamma=$ $\gamma\left(\lambda_{0}, T\right)$ is the rank of $\lambda_{0}, k_{n} \geq 1$ is the number of the different eigenvalues 
$\lambda_{n}^{(1)}, \ldots, \lambda_{n}^{\left(k_{n}\right)}$ of $P_{n} T$ in $\Lambda_{0}, \bar{\lambda}_{n}$ is the average of $\lambda_{n}^{(i)}\left(i=1, \ldots, k_{n}\right)$ weighted by their algebraic multiplicities $\mu\left(\lambda_{n}^{(i)}, P_{n} T\right)=\operatorname{dim} W\left(\lambda_{n}^{(i)}, P_{n} T\right)$,

$$
\bar{\lambda}_{n}=\left[\sum_{i=1}^{k_{n}} \mu\left(\lambda_{n}^{(i)}, P_{n} T\right) \lambda_{n}^{(i)}\right]\left[\sum_{i=1}^{k_{n}} \mu\left(\lambda_{n}^{(i)}, P_{n} T\right)\right]^{-1}
$$

and $\delta_{n}$ is defined by (2.11) or (2.12), corresponding to the quasi-uniform grid $\{(2.1),(2.2)\}$ or the graded grid $\{(2.1),(2.3)\}$, respectively.

Proof. On the basis of the convergence (3.4) it follows from the results of [8; $9 ; 17 ; 19]$ that

$$
\left|\lambda_{n}-\lambda_{0}\right| \leq c^{\prime} \theta_{n}^{1 / \gamma}, \quad\left|\lambda_{n}-\lambda_{0}\right| \leq c^{\prime \prime} \theta_{n}^{1 / k_{n}}, \quad\left|\bar{\lambda}_{n}-\lambda_{0}\right| \leq c^{\prime \prime \prime} \theta_{n},
$$

with some positive constants $c^{\prime}, c^{\prime \prime}$ and $c^{\prime \prime \prime}$ not depending on $n$, and

$$
\theta_{n}=\sup \left\{\left\|y-P_{n} y\right\|_{C[a, b]}: y \in W\left(\lambda_{0}, T\right),\|y\|_{C[a, b]}=1\right\}, n \in \mathbb{N} .
$$

Using Theorem 1.1 with $f=0$ (see Remark 1.1) and induction by $i$, we conclude that $N\left(\left(\lambda_{0} I-T\right)^{i}\right) \subset C^{3, \beta}[a, b]$ for $i=1,2, \ldots$ Therefore, $W\left(\lambda_{0}, T\right) \subset$ $C^{3, \beta}[a, b], 0<\beta<3, \lambda_{0} \neq 0$, and we obtain from (3.3) and (3.7) that

$$
\theta_{n} \leq c \delta_{n}, \quad n \in \mathbb{N}
$$

where $c$ is a positive constant not depending on $n$. The estimates (3.5) now follow from $(3.6)-(3.8)$.

\section{NUMERICAL EXAMPLES}

We present two examples.

Example 4.1. Consider the integral equation

$$
y(t)=\int_{0}^{1}|t-s|^{\frac{3}{2}} y(s) d s+f(t), \quad 0 \leq t \leq 1,
$$

where

$$
\begin{array}{r}
f(t)=t^{\frac{5}{2}}-\frac{3 \pi}{256} t^{5}-\frac{1}{5} \sqrt{1-t}+\frac{11}{40} t \sqrt{1-t}-\frac{1}{80} t^{2} \sqrt{1-t}-\frac{1}{64} t^{3} \sqrt{1-t} \\
-\frac{3}{128} t^{4} \sqrt{1-t}-\frac{3}{256} t^{5} \ln (2-t+2 \sqrt{1-t})+\frac{3}{256} t^{5} \ln (t) .
\end{array}
$$

It is easy to check that $y(t)=t^{5 / 2}$ is the exact solution of equation (4.1), $f \in$ $C^{3, \frac{1}{2}}[0,1]$, and the assumptions (1.2) and (1.3) are fulfilled with $g(t, s)=1$, $\kappa(t-s)=|t-s|^{3 / 2}$ and $\beta=1 / 2$. 
Example 4.2. Consider the integral equation

$$
y(t)=\int_{0}^{1}|t-s|^{-\frac{1}{2}} y(s) d s+f(t), \quad 0 \leq t \leq 1,
$$

where

$$
\begin{aligned}
f(t)=\sqrt{t}-\frac{\pi}{2} t-\frac{(1+\sqrt{1-t})^{2}}{4}+\frac{t^{2}}{4(1+\sqrt{1-t})^{2}} & \\
& -t \ln (1+\sqrt{1-t})+\frac{t \ln (t)}{2} .
\end{aligned}
$$

It is easy to check that $y(t)=\sqrt{t}$ is the exact solution of equation (4.2), $f \in C^{3, \frac{5}{2}}[0,1]$ and the assumptions (1.1) and (1.3) are fulfilled with $g(t, s)=1$, $\kappa(t-s)=|t-s|^{-1 / 2}$ and $\beta=5 / 2$.

For the numerical solution of equations (4.1) and (4.2) let $n \geq 4$ be a natural number or an even natural number depending whether we use the quasiuniform grid or the graded grid. Let $0=t_{0}<t_{1}<\ldots<t_{n}=1$ be a partition of the interval $[a, b]=[0,1]$ satisfying the condition $(2.2)$ with some constant $q \geq 1$ independent of $n$ (in case of the quasi-uniform grid) or let the grid points $t_{0}, t_{1}, \ldots, t_{n}$ be given by the formulas (2.3) with $a=0, b=1$ and some fixed real number $r \geq 1$ not depending on $n$ (in case of the graded grid). We define the interpolation points $x_{i}, i=0, \ldots, n+1$, by the formulas (2.5), where we take $\eta=1 / 2$. Under these assumptions we solved the equations (4.1) and (4.2) numerically by the collocation method (2.6) using the presentation (2.7) and computing exactly the integrals

$$
\int_{0}^{1} K\left(x_{i}, s\right) y_{n}(s) d s, \quad i=0, \ldots, n+1,
$$

with $K(t, s)=\kappa(t-s)$.

For the estimation of the error $\left\|y_{n}-y\right\|_{C[0,1]}$ we also introduce another partition of the interval $[0,1]$ with the grid points $\tau_{i j}$, namely

$$
\tau_{i j}=t_{i}+\frac{t_{i+1}-t_{i}}{10} j, \quad i=0, \ldots, n-1, j=0, \ldots 10 .
$$

In the following Tables $1-4$ the estimations $\varepsilon_{n}$ of the norm $\left\|y_{n}-y\right\|_{C[0,1]}$,

$$
\varepsilon_{n}=\max _{\substack{0 \leq i \leq n-1 \\ 0 \leq j \leq 10}}\left|y_{n}\left(\tau_{i j}\right)-y\left(\tau_{i j}\right)\right|
$$


Table 1 .

Eq. (4.1), quasi-uniform grid

\begin{tabular}{c|c|c|c|c|c|c}
\hline \multirow{2}{*}{$n$} & \multicolumn{2}{|c|}{$q=1$} & \multicolumn{4}{c}{$q=4$} \\
\cline { 2 - 7 } & $\varepsilon_{n}$ & $\rho_{n}$ & $\varepsilon_{n}$ & $\rho_{n}$ & $\varepsilon_{n}$ & $\rho_{n}$ \\
\hline 4 & 0.0001470 & & 0.0004670 & & 0.0003907 & \\
8 & 0.0000259 & 5.675 & 0.0000839 & 5.565 & 0.0000551 & 7.091 \\
16 & 0.0000045 & 5.667 & 0.0000148 & 5.666 & 0.0000073 & 7.452 \\
32 & 0.0000008 & 5.668 & 0.0000026 & 5.657 & 0.0000009 & 7.703 \\
64 & 0.0000001 & 5.671 & 0.0000004 & 5.685 & 0.0000001 & 7.828 \\
128 & 0.0000000 & 5.667 & 0.0000000 & 5.618 & 0.0000000 & 7.965 \\
\hline
\end{tabular}

Table 2.

Eq. (4.1), graded grid

\begin{tabular}{c|c|c|c|c|c|c}
\hline \multirow{2}{*}{$n$} & \multicolumn{2}{|c|}{$r=1$} & \multicolumn{2}{c|}{$r=1.2$} & \multicolumn{2}{c}{$r=3$} \\
\cline { 2 - 7 } & $\varepsilon_{n}$ & $\rho_{n}$ & $\varepsilon_{n}$ & $\rho_{n}$ & $\varepsilon_{n}$ & $\rho_{n}$ \\
\hline 4 & 0.0001470 & & 0.0000754 & & 0.0008757 & \\
8 & 0.0000259 & 5.675 & 0.0000094 & 8.009 & 0.0001330 & 6.583 \\
16 & 0.0000045 & 5.667 & 0.0000011 & 7.939 & 0.0000173 & 7.649 \\
32 & 0.0000008 & 5.668 & 0.0000001 & 8.002 & 0.0000021 & 7.937 \\
64 & 0.0000001 & 5.671 & 0.0000000 & 7.925 & 0.0000002 & 8.002 \\
128 & 0.0000000 & 5.667 & 0.0000000 & 5.755 & 0.0000000 & 7.954 \\
\hline
\end{tabular}

and the ratios

$$
\rho_{n}=\varepsilon_{n / 2} / \varepsilon_{n}
$$

which characterize the rate of convergence of the method (2.6) are presented for both the quasi-uniform and the graded grid.

In case of the quasi-uniform grid we have given the convergence results for two values of the parameter $q$ (see condition (2.2)): $q=1$ and $q=4$. Notice also that for $q=4$ we have constructed the grid in two ways. Namely, in the first case for $n=4$ we have chosen the grid points $t_{0}=0, t_{1}=0.4, t_{2}=$ $0.5, t_{3}=0.6, t_{4}=1$, in the second case we have selected the grid points $t_{0}=0, t_{1}=0.1, t_{2}=0.5, t_{3}=0.9, t_{4}=1$, and in both cases for every other $n=8,16, \ldots$ the new grid was obtained by taking the old grid points for $n / 2$ and the centerpoints of the subintervals corresponding to the partition of the interval $[0,1]$ for $n / 2$.

In case of the graded grid we have presented the convergence results for three values of the parameter $r$ (see formulas (2.3)): in case of equation (4.1) we have taken $r=1, r=1.2$ and $r=3$, and in case of equation (4.2) $r=1$, $r=3$ and $r=6$.

From Theorem 2.1 we can derive the following convergence results: for equation (4.1) we have $\beta=1 / 2$ and then in case of the quasi-uniform grid the ratio $\rho_{n}$ must be $2^{5 / 2} \approx 5.657$, and in case of the graded grid the ratio $\rho_{n}$ must be $2^{5 / 2} \approx 5.657,2^{3}=8$ and $2^{3}=8$ for $r=1, r=1.2$ and $r=3$, respectively. For equation (4.2) we have $\beta=5 / 2$ and then in case of the quasi-uniform grid the ratio $\rho_{n}$ must be approximately $\sqrt{2} \approx 1.414$ and in case of the graded grid 
Table 3.

Eq. (4.2), quasi-uniform grid

\begin{tabular}{c|c|c|c|c|c|c}
\hline \multirow{2}{*}{$n$} & \multicolumn{2}{|c|}{$q=1$} & \multicolumn{4}{c}{$q=4$} \\
\cline { 2 - 7 } & $\varepsilon_{n}$ & $\rho_{n}$ & $\varepsilon_{n}$ & $\rho_{n}$ & $\varepsilon_{n}$ & $\rho_{n}$ \\
\hline 4 & 0.0681131 & & 0.0967526 & & 0.0393239 & \\
8 & 0.0433667 & 1.571 & 0.0584728 & 1.655 & 0.0256411 & 1.534 \\
16 & 0.0289248 & 1.499 & 0.0379341 & 1.541 & 0.0174917 & 1.466 \\
32 & 0.0197214 & 1.467 & 0.0255248 & 1.486 & 0.0121019 & 1.445 \\
64 & 0.0136114 & 1.449 & 0.0174838 & 1.460 & 0.0084330 & 1.435 \\
128 & 0.0094672 & 1.438 & 0.0121015 & 1.445 & 0.0059033 & 1.429 \\
\hline
\end{tabular}

Table 4.

Eq. (4.2), graded grid

\begin{tabular}{c|c|c|c|c|c|c}
\hline \multirow{2}{*}{$n$} & \multicolumn{2}{|c|}{$r=1$} & \multicolumn{2}{c|}{$r=3$} & \multicolumn{2}{c}{$r=6$} \\
\cline { 2 - 7 } & $\varepsilon_{n}$ & $\rho_{n}$ & $\varepsilon_{n}$ & $\rho_{n}$ & $\varepsilon_{n}$ & $\rho_{n}$ \\
\hline \multirow{2}{*}{4} & 0.0681131 & & 0.0141109 & & 0.0109106 & \\
8 & 0.0433667 & 1.571 & 0.0046903 & 3.008 & 0.0018694 & 5.836 \\
16 & 0.0289248 & 1.499 & 0.0016290 & 2.879 & 0.0001951 & 9.582 \\
32 & 0.0197214 & 1.467 & 0.0005733 & 2.841 & 0.0115118 & 0.017 \\
64 & 0.0136114 & 1.449 & 0.0002024 & 2.832 & 2.0648866 & 0.006 \\
128 & 0.0094672 & 1.438 & 0.0000715 & 2.830 & 2.9964757 & 0.689 \\
\hline
\end{tabular}

the ratio $\rho_{n}$ must be $\sqrt{2} \approx 1.414,2^{3 / 2} \approx 2.828$ and $2^{3}=8$ for $r=1, r=3$ and $r=6$, respectively. Notice also that in case of the graded grid the values $r=1.2$ (also $r=3$ ) for $\beta=1 / 2$ and $r=6$ for $\beta=5 / 2$ correspond to the best possible rate of global convergence, the third order convergence of the method $(2.6)$ (see $\{(2.10),(2.12)\}$ ).

From the Tables $1-4$ we can see that the numerical results are quite well in accord with theoretical estimations except in Table 4 where in case $r=6$ we have the loss of accuracy: the error for $n$ is bigger than the error for $n / 2$ if $n=32, n=64$ and $n=128$. This shows that practical application of the method (2.6) can lead to extra complications if dealing with weakly singular integral equations with sufficiently strong singularity in kernel (like equation (4.2)). The numerical results also show that if we construct the quasi-uniform grid "wisely", i.e. if the grid is more dense near the endpoints 0 and 1 of the domain of integration, then we can obtain almost the best possible rate of convergence, the third order convergence (see the last column of Table 1).

\section{REFERENCES}

[1] K. Atkinson. The Numerical Solution of Integral Equations of the Second Kind. Cambridge University Press, 1997.

[2] C.T.H. Baker. The Numerical Treatment of Integral Equations. Claredan Press, Oxford, 1977.

[3] H. Brunner, A. Pedas and G. Vainikko. The piecewise polynomial collocation method for 
nonlinear weakly singular Volterra equations. Math. Comput., 68(227), 1079 - 1095, 1999.

[4] W. Hackbusch. Integralgleichungen Theorie und Numerik. Teubner, Stuttgart, 1989.

[5] K. Hakk, O. Karma and A. Pedas. Collocation method for weakly singular integral equation and corresponding eigenvalue problem. In: Mathematical Research. 5, Tools for Mathematical Modelling, Proceedings of the 2nd International Conference: Tools for Mathematical Modelling, Saint-Petersburg, June 14-19, 1999. Saint-Petersburg State Technical University Press, 36 - 45, 1999.

[6] H. Kaneko, R. Noren and Y. Xu. Numerical solutions for weakly singular Hammerstein equations and their superconvergence. J. Integral Equations Appl., 4, 391 - 406, 1992.

[7] R. Kangro, R. Pallav and A. Pedas. Quadratic spline collocation method for weakly singular integral equations. Proc. Estonian Acad. Sci. Phys. Math., 51(1), 47 - 60, 2002.

[8] O. Karma. Approximation in eigenvalue problems for holomorphic Fredholm operator functions I. Numer. Funct. Anal. and Optimiz., 17(3\&4), 365 - 387, 1996.

[9] O. Karma. Approximation in eigenvalue problems for holomorphic Fredholm operator functions II. Numer. Funct. Anal. and Optimiz., 17(3\&4), 389 - 408, 1996.

[10] M.A. Krasnoselski, G.M. Vainikko, P.R. Zabreiko, J.B. Rutitskii and V.J. Stetsenko. Approximate Solution of Operator Equations. Nauko, Moscow, 1969. (in Russian)

[11] G. Monegato and L. Scuderi. High order methods for weakly singular integral equations with nonsmooth input functions. Math. Comput., 67(224), 1493 - 1515, 1998.

[12] R. Pallav. On the quadratic spline collocation method for weakly singular integral equations. In: Abstracts of the $7^{\text {th }}$ International Conference MMA2002, May 31-June 2, 2002, Kääriku, Estonia, 43. Estonian Mathematical Society, 2002.

[13] R. Pallav. Quadratic spline collocation method for weakly singular integral equations on graded grids. Proc. Estonian Acad. Sci. Phys. Math., 51(4), 2002.

[14] A. Pedas and G. Vainikko. Superconvergence of piecewise polynomial collocations for nonlinear weakly singular integral equations. J. Integral Equations Appl., 9, 379 - 406, 1997.

[15] Y. Savyalov, B. Kvasov and V. Miroshnichenko. The Spline-Function Method. Nauka, Moscow, 1980. (in Russian)

[16] E. Tamme. Fully discrete collocation method for weakly singular integral equations. Proc. Estonian Acad. Sci. Phys. Math., 50(3), 133 - 144, 2001.

[17] G. Vainikko. Analysis of Discretization Methods. University of Tartu, Tartu, 1976. (in Russian)

[18] G. Vainikko. Multidimensional Weakly Singular Equations. Lecture Notes in Math., 1549. Springer, 1993. (in Russian)

[19] G. Vainikko, A. Pedas and P. Uba. Methods for Weakly Singular Integral Equations. University of Tartu, Tartu, 1984. (in Russian)

\section{Silpnai singuliarių integralinių lygčių ir tikrinių reikšmių uždavinio sprendimas kvadratiniu kolokacinių splainų metodu}

R. Pallav, A. Pedas

Darbe sprendžiama silpnai netiesinè antrojo tipo Fredholmo lygtis ir atitinkamas tikriniu reikšmiu uždavinys. Naudojami kvazi-pastovus ir specialiai sutankejjantys tinklai. Ištirtas gautų diskretizacijų konvergavimo greitis, parodyta, kuriais atvejais šis konvergavimas yra optimalus. Pateikti skaitinio eksperimento rezultatai, patvirtinantys teorinius rezultatus. 